Rhode Island College

Digital Commons @ RIC

$5-5-2021$

\title{
Knowledge of Electrolyte Replacement Protocols Among Medical- Surgical Registered Nurses: A Research Project
}

\author{
Sarah-Jane H. Santos
}

Follow this and additional works at: https://digitalcommons.ric.edu/etd

Part of the Nursing Commons

\section{Recommended Citation}

Santos, Sarah-Jane H., "Knowledge of Electrolyte Replacement Protocols Among Medical-Surgical Registered Nurses: A Research Project" (2021). Master's Theses, Dissertations, Graduate Research and Major Papers Overview. 388.

https://digitalcommons.ric.edu/etd/388

This Major Paper is brought to you for free and open access by the Master's Theses, Dissertations, Graduate Research and Major Papers at Digital Commons @ RIC. It has been accepted for inclusion in Master's Theses, Dissertations, Graduate Research and Major Papers Overview by an authorized administrator of Digital Commons @ RIC. For more information, please contact digitalcommons@ric.edu. 
Knowledge of Electrolyte Replacement Protocols Among Medical-Surgical Registered Nurses: A Research Project

by

Sarah-Jane H. Santos, BSN, RN-BC

A Major Paper Submitted in Partial Fulfillment

of the Requirements for the Degree of

Master of Science in Nursing

in

The School of Nursing

Rhode Island College

2021 


\begin{abstract}
Electrolyte abnormalities in hospitalized patients may result from a variety of acute and chronic medical conditions. Hospital admissions related to electrolyte imbalances in adult patients may be a direct result of acute illness such as nausea, vomiting, diarrhea, and dehydration or complications related to medications or surgical procedures. Patients with deficiencies of electrolytes such as potassium, magnesium and phosphorus are at an increased risk for life threatening complications such as seizures, delirium, cardiac dysrhythmias, respiratory muscle weakness and respiratory compromise. Nurses must possess a baseline knowledge of electrolyte disturbances and recognize potential and possible complications related to deficiencies of key electrolytes. The purpose of this research project was to improve nurses' knowledge regarding the management, treatment, and care of adult patients with electrolyte imbalances. An educational intervention was developed and a pre-posttest design was utilized to evaluate the effect of the intervention. This research project demonstrated that the implementation of an educational intervention increased nurses' knowledge regarding the management, treatment, and care of adult patients with electrolyte abnormalities. The results of this research project validated the need for additional education for nurses which focuses on the care of adult patients with electrolyte deficiencies.
\end{abstract}




\section{Acknowledgements}

There were many people instrumental in the accomplishment of this project. Thank you to my first reader, Dr. Sharon Galloway, for her time, commitment, and guidance throughout the entire process of this research project. Also, thank you to my second reader Dr. Joanne Costello for her time and input in this major paper. I would also like to thank my mentor at the Providence VA Medical Center, Mackenzie Glashow, and my unit manager, Ashley Spellman, for their help and support during the development and implementation of this project. To my friend Brooke Maurice, thank you for being there with me through every class, every exam, and everything in between over the past three years. I don't think I would have made it through the program without your continuous support. Finally, thank you to my husband, Peter, for his unwavering support and encouragement over the last three years in seeing I achieved this goal. 


\section{Table of Contents}

Background/Statement of the Problem......................................... 1

Literature Review........................................................ 3

Theoretical Framework........................................................... 17

Method..................................................................... 20

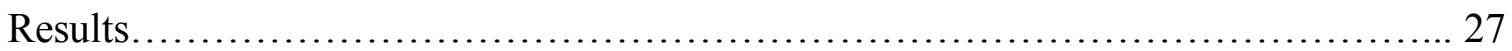

Summary and Conclusions...................................................... 31

Recommendations and Implications for Advanced Nursing Practice................. 35

References................................................................. 38

Appendices............................................................. 42 
Knowledge of Electrolyte Replacement Protocols Among Medical-Surgical Registered

Nurses: A Research Project

\section{Background/Statement of the Problem}

Electrolyte imbalances are common among hospitalized patients, which can lead to adverse effects and poor outcomes for the acute care population. In particular, deficiencies in potassium, magnesium, and phosphorus are common among patients seen in the emergency room and inpatient units. The presence of electrolyte abnormalities leads to increased emergency room visits, increases hospital readmission rates and is associated with an increased 30-day and one-year mortality rates (Tazmini, 2019). In some instances, the electrolyte imbalances can be a contributing factor to inpatient admission diagnosis such as dehydration, malnutrition, or renal failure. In contrast, there are patients that develop electrolyte imbalances independent of their acute diagnoses. In both instances, the electrolyte imbalances should be corrected to prevent further complications such as fatigue, lethargy, confusion, renal injury, respiratory failure, and death. Deficiencies in potassium and magnesium are associated with increased risk for ventricular tachycardia and other arrythmias (Pearson, 2018). In patients admitted to a critical care unit, hypomagnesaemia was associated with mortality rates twice as high as comparably ill normomagnesemia patients (Wolf \& Hilewitz, 2014). In cases of mild to moderate hypokalemia research has shown increased morbidity and mortality up to $50 \%$ for patients with cardiovascular and kidney disease (Jensen et al., 2015).

In some institutions, standardized electrolyte replacement protocols are used for many diagnoses excluding patients with multiple comorbidities such as kidney disease, diabetes and heart failure. According to Todd et al. (2009), protocol-driven care allows 
clinicians to complement their clinical judgement with the latest evidence to improve patient outcomes. The use of standardized electrolyte replacement protocols for patients, according to Pearson et al. (2018), can significantly decrease the time frame between abnormal laboratory results and the administration of electrolyte replacement. The protocols decrease the need for nurses to seek orders from a provider before providing necessary electrolyte replacement. The decreased time between the laboratory results and the administration of electrolyte replacement has shown to help prevent complications and adverse outcomes for patients by preventing further deficiencies of vital electrolytes. The implementation of evidence-based medicine guidelines for electrolyte replacement can also provide a method of consistent therapy throughout the facility (MacLaren et al, 1999). The use of standardized electrolyte replacement protocols may also increase autonomy for nursing staff and decrease workload for physicians (Pearson et al, 2018). The purpose of this research project is to determine whether the implementation of an electrolyte replacement educational program will improve nurse's knowledge related to the management, treatment, and care of adult patients with electrolyte imbalances.

In the next section, a review of the literature will be discussed. 


\section{Literature Review}

A literature review was conducted utilizing the search engines CINAHL, PubMed, and Medline. Literature was searched from the year 2010 until the present and was limited to journals published in English. The following key words were used: potassium, magnesium, phosphorus, electrolytes, electrolyte replacement, and electrolyte deficiency. Additional searches were done using the terms hypokalemia, hypomagnesaemia, hypophosphatemia, electrolyte repletion, and electrolyte replacement protocols. The key words and terms were searched individually and/or in combination. Search results were limited to the specific electrolytes including potassium, magnesium and phosphorus, and the inpatient hospital.

\section{Electrolyte Basics}

Potassium. Potassium is the second most prevalent intracellular cation and the most abundant cation within the intracellular fluid. Potassium plays a key role in maintaining normal cell function using a pump called the Na+-K+-ATPase pump (E. Kardalas, et al., 2018). The constant movement of potassium across the cell membranes is based on the needs of the body, creating an action potential which many tissues rely on for function such as nerve conduction, muscle contraction and kidney function. Enzyme activities along with cell division and growth are catalyzed by potassium and are affected by any alterations in its concentration (E. Kardalas, et al., 2018). The majority of potassium in the human body is held within the cells in the intracellular fluid. The amount of potassium in the extracellular fluid is significantly less, with normal levels measuring between $3.5-5 \mathrm{mEq} / \mathrm{L}$ (Norris, 2019). Potassium is absorbed in the small intestines and used by the body to maintain intercellular and extracellular function. 
Excretion of potassium is controlled by the kidneys with a majority of potassium being excreted in urine, and the remaining excreted in stool and sweat. Adequate intake of potassium is necessary for normal cell function within the body.

Magnesium. Approximately $99 \%$ of the magnesium in the body is stored in the bone, muscles and soft tissue, with 1\% remaining in the extracellular fluid (Ayuk \& Gittoes, 2011). Magnesium is an intercellular cation, second only to potassium within the intracellular fluid (Wolf \& Hilewitz, 2014). This intercellular cation plays a role in bodily functions such as carbohydrate metabolism and protein synthesis. Magnesium, a mineral in the body, is responsible for many chemical reactions, muscle and nerve function, blood glucose control, and blood pressure regulation (National Institute of Health [NIH], 2019). Magnesium also plays a role in the active transport of calcium and potassium ions across cell membranes, a process that is important to nerve impulse conduction, muscle contraction, and normal heart rhythms (NIH, 2019). Normal magnesium concentrations in adults range from 1.3-2.1 mEq/L (Norris, 2019). Magnesium is absorbed in the intestines, however only $25-65 \%$ of dietary magnesium is absorbed. Regulation of magnesium reabsorption and excretion is controlled by the kidneys, with excretion in the urine. Magnesium concentration affects other electrolyte functions within the human body which can impact the clinical course of the inpatient population.

Phosphorus. Phosphorus is an intracellular anion and is essential to many bodily functions. Eight-five percent of phosphate is found in the bones and teeth, $14 \%$ is found in the soft tissues, and 1\% in the extracellular fluid (Kear, 2017). Phosphorus plays key roles in regulation of gene transcription, activation of enzymes, maintenance of normal 
pH in extracellular fluid, and intracellular energy storage (NIH, 2019). The normal levels of phosphorus within the adult human body are $2.5-4.5 \mathrm{mg} / \mathrm{dL}$, levels are slightly higher in children and infants related to their increased growth hormones (NIH, 2019). Phosphorus is ingested by the foods we eat, mainly milk and meat products. Phosphorus is absorbed in the intestines, primarily the jejunum. However, the absorption of phosphorus can be negatively affected by ingestion of other substances such as calcium, magnesium, and aluminum (Norris, 2019). The kidneys, bones, and intestines regulate phosphate levels, excreting excess phosphate though urine based on the total concentration within the body.

\section{Electrolyte Imbalances}

Hypokalemia. According to the NIH (2019), hypokalemia is defined as a serum potassium level less than $3.5 \mathrm{mmol} / \mathrm{L}$, which is a common occurrence among hospitalized patients for a myriad of reasons. Potassium deficiency, also known as hypokalemia, has the potential to cause weakness, hypertension, muscle cramps and can progress to respiratory distress and lethal cardiac arrhythmias. Some of the more common causes of hypokalemia in hospitalized patients include kidney injury or disease, illegal drugs, medications, gastrointestinal losses, cellular shifts, sweating, and decreased nutritional intake (Kear, 2017). In study by Hemstreet et al. (2006), out of 58,000 hospitalized patients evaluated over the course of three years, $20 \%$ of patients had a serum potassium of less than $3.5 \mathrm{mEq} / \mathrm{L}$ during their hospital admission. Decreased levels of potassium can cause paralysis, rhabdomyolysis, and myocardial infarction, and has been associated with difficulty in weaning patients from mechanical ventilation (Scotto et al., 2014). Hypokalemia in hospitalized patients is common and is associated with an increased 7- 
day mortality rate compared to hospitalized patients with levels of potassium within the appropriate range (Jensen et al., 2015). A study by Jensen et al. (2015) suggests that patients with hypokalemia who survive the first seven days after hospital admission have an increased rate of mortality starting on day eight through day thirty. More than $50 \%$ of patients with clinically significant hypokalemia also have a concomitant magnesium deficiency which is most frequently observed in individuals requiring loop or thiazide diuretic therapy (E. Kardalas et al., 2018). The symptoms related to hypokalemia directly relate to the severity of the potassium deficiency. The presence of electrolyte abnormalities including hypokalemia contributes to increased hospital length of stay, readmission rates, in-hospital thirty day, and one-year mortality rates (Tazmini et al., 2019).

Hypomagnesaemia. Hypomagnesaemia, or low magnesium levels, is relatively common, with an estimated prevalence of $2.5-15 \%$ in the general population, however prevalence increases up to $65 \%$ with patients admitted to the hospital and in intensive care units (Ayuk \& Gittoes, 2011). A low plasma concentration of magnesium indicates a systemic lack of magnesium. Drug induced hypomagnesaemia, particularly related to the use of proton pump inhibitors (PPI) is becoming increasingly more common (Ayuk \& Gittoes, 2011). This electrolyte deficiency occurs in many patients with multiple significant comorbidities such as congestive heart failure, renal failure, and diabetes mellitus, and contributes to higher mortality and longer hospital stays (Wolf \& Hilewitz, 2014). According to a study by Wolf and Hilewitz (2014), hypomagnesaemia has been linked to incidences of atrial fibrillation in patients without cardiovascular disease and is associated with increased risk of metabolic syndrome, hypertension, diabetes, and 
hyperlipidemia. According to the National Institute of Health, hypomagnesaemia which is defined as a plasma concentration of magnesium less than $1.8 \mathrm{mg} / \mathrm{dL}$. Deficiencies in magnesium can be the result of gastrointestinal losses, alcoholism, refeeding syndrome, and multiple medications (Kear, 2017). Magnesium and albumin have an associated relationship, low serum albumin levels result in low levels of magnesium which can disrupt the fluid homeostasis of the body and lead to further complications for the patient (Kear, 2017). Magnesium deficiency commonly occurs with hypokalemia and have similar neurological and cardiovascular effects including tachycardia, hypertension, ventricular dysrhythmias, personality changes, and muscle tremors (Norris, 2019).

Hypophosphatemia. Deficiency in the electrolyte phosphorus or hypophosphatemia, is defined as a plasma concentration less than $2.7 \mathrm{mg} / \mathrm{dL}$, is an electrolyte disorder that is frequently common in hospitalized patients (Hemstreet, 2006). Phosphorus circulating in the bloodstream in present in relatively low levels, therefore significant decreases in serum values can have serious physiologic effects on cardiac, respiratory, and muscle function (Kear, 2017). Phosphate levels can be affected by alcoholism, burns, kidney disease, and herbal supplements, as well as medications such as antacids and laxatives (Kear, 2017). Hypophosphatemia is frequently caused by hyperparathyroidism, renal tubule defects, and diabetic ketoacidosis (NIH, 2019). Hypophosphatemia can cause blood disorders such as anemia, as well as neurological and musculoskeletal symptoms (Norris, 2019). Clinical manifestations of phosphate deficiency include tremor, ataxia, confusion, seizures, bone pain and muscle weakness. Phosphate deficiencies are typical complications of chronic kidney disease and cardiovascular disease. 


\section{Management of Electrolyte Replacement}

Many patients require electrolyte replacement while hospitalized and the process of electrolyte replacement varies by facility. Some processes are provider driven based on daily labs values while others are more nurse driven using standardized electrolyte replacement protocols. In general, protocol driven care allows clinicians to use patient presentation and laboratory data supported by their clinical judgement and best practice to improve patient outcomes (Todd et al.,2009). Numerous research studies support the use of standard protocols to improve patient care. Examples of current and successful evidence-based protocols include transfusion of blood products and the treatment of patients with sepsis and strokes. The results of previous studies (Couture et al., 2013 \& Zielenski et al., 2017) have suggested that the use of standardized electrolyte repletion protocols is more effective than typically used approaches to electrolyte repletion. According to Zielenski et al. (2017), superiority of nurse driven electrolyte replacement has been demonstrated over physician ordered electrolyte replacement practices in hospitalized patients. The use of standardized electrolyte replacement protocols is increasing and can help eliminate variations in care such as clinician preference, timing, and dosing. Protocols should be developed by interdisciplinary teams and are based on evidence-based practice.

\section{Current Replacement Recommendations}

The recommended serum levels of phosphorus are between $2.8-4.5 \mathrm{mEq} / \mathrm{dL}$ in adult patients and should be replaced if levels reach $2.7 \mathrm{mEq} / \mathrm{dL}$ or below. According to Hemstreet et al. (2006), patient with severe hypophosphatemia are candidates for intravenous phosphate repletion therapy. According to the NIH (2019), the normal serum 
concentration of potassium is 3.6 to $5.0 \mathrm{mmol} / \mathrm{L}$, and replacement is recommended when levels decrease to below $3.4 \mathrm{mmol} / \mathrm{L}$. The patient's medical history, age, weight, urine output, Blood Urea Nitrogen (BUN) and Creatinine (Cr) should be considered prior to the administration of potassium. The NIH reports that the normal serum magnesium level is between 1.5-2.5 mEq/L, and replacement should be considered when levels decrease below $1.4 \mathrm{mEq} / \mathrm{L}$. According to Ayuk and Gittoes (2011), symptomatic hypomagnesaemia should be treated with intravenous magnesium reserving oral magnesium for asymptomatic patients.

\section{Electrolyte Replacement Protocol}

The literature provides examples of best practice electrolyte replacement and standard protocols that have been implemented in practice. According to Todd et al. (2009), a recommendation exists that includes the following potassium replacement protocol: potassium levels between $3.8-5 \mathrm{mEq} / \mathrm{L}$, no replacement is needed; levels between 3.5-3.7 mEq/L provide $40 \mathrm{mEq}$ orally, levels between 3.1-3.4 $\mathrm{mEq} / \mathrm{L}$ provide 60 $\mathrm{mEq}$; give potassium orally or intravenously, however, orally is preferred; recheck potassium levels with the next morning lab draw; if the patient's potassium level is less than 3.1, the patient will be given $80 \mathrm{mEq}$ orally or intravenously, and the level checked four hours after administration of replacement. The protocol for magnesium replacement suggested by Todd et al. (2009) includes: levels between 1.9-2.7 mEq/L, no replacement needed; levels between $1.5-1.8 \mathrm{mEq} / \mathrm{L}$ provide $2 \mathrm{~g}$ intravenously or $1600 \mathrm{mg}$ orally; levels between 1.1-1.4 mEq/L provide $4 \mathrm{~g}$ intravenously or $3200 \mathrm{mg}$ orally; give magnesium orally or intravenously, however, oral is preferred; check magnesium levels with next morning lab draw; if patients level is below $1.1 \mathrm{mEq} / \mathrm{L}$ provide $6 \mathrm{~g}$ intravenously or 
$4800 \mathrm{mg}$ orally; and the level checked four hours after administration of replacement. No more than $800 \mathrm{mg}$ of oral magnesium should be given at one time, with doses spread out by 1-2 hours. The protocol for phosphorus replacement suggested by Todd et al. (2009) includes: levels between $2.5-4.8 \mathrm{mg} / \mathrm{dL}$ no replacement required, levels between 1.8-2.4 $\mathrm{mg} / \mathrm{dL}$ provide $20 \mathrm{mM}$ intravenously; levels between $1.0-1.7$ provide $30 \mathrm{mM}$ intravenously; check phosphorus level with the next morning lab draw; levels less than $1.0 \mathrm{mg} / \mathrm{dL}$ provide 40mM intravenously; level checked four hours after administration of replacement. The protocol suggests that all phosphate intravenous replacement be infused over six to eight hours. The protocol is not intended for patients with end-stage renal disease (ESRD), acute renal failure (ARF) or insufficiency, chronic heart failure, diabetic ketoacidosis (DKA), or pregnancy induced hypertension (Todd et al., 2009). Zielenski et al. (2017) demonstrated the use of a timed, electronic, assessment driven potassium replacement protocol (TARP) may be responsible for improved patient outcomes. The TARP requirements include: draw a potassium level at 0600 and 1800 and assess laboratory results at 0800 and 2000. If the patient is able to tolerate oral medications the medications should be given orally, otherwise intravenous will be used. If a patients potassium level is less than $3.2 \mathrm{mEq} / \mathrm{L}$ they will receive $40 \mathrm{mEq}$ once, levels between 3.3-3.4 mEq/L 30mEq once, and between 3.5-3.6 mEq/L 20mEq is administered. In contrast to the previous protocol discussed, level between 3.7-3.9 $\mathrm{mEq} / \mathrm{L}$, patients receive a onetime dose of $10 \mathrm{mEq}$, and no replacement is required for levels greater than $3.9 \mathrm{mEq} / \mathrm{L}$. For potassium levels less than or equal to 3.2 , the replacement will be administered and the provider will be notified. The protocol does not go into detail regarding any specific patient exclusion criteria. 
Pearson et al. (2018) designed an electrolyte replacement protocol with specific inclusion criteria for use in the inpatient setting. Exclusion criteria was also established and included benchmarks such as Glomerular Filtration Rate (GFR) less than 30, Total Parenteral Nutrition (TPN) infusion, patients with DKA, urine output less than $15 \mathrm{ml} / \mathrm{hr}$, and continuous renal replacement therapy (CRRT).

\section{Benefits of Electrolyte Replacement Protocols}

In a retrospective study by Todd et al. (2009), a 27-bed surgical intensive care unit was used to compare patient's electrolyte concentrations before and after implementing a standardized replacement protocol. Prior the implementation of the electrolyte replacement protocol on the unit, providers would complete morning rounds, patient assessments, and review of pertinent labs, then required electrolyte replacement would be ordered and administered. After implementation of the electrolyte replacement protocol on the unit, the nurse was responsible for reviewing the lab work and providing electrolyte replacement based on a standard protocol. The findings of the study indicated a $10 \%$ increase in electrolyte replacement and a $15 \%$ increase in overall effectiveness of treatment (Todd et al., 2009). The researchers also reported an improvement in provider satisfaction and autonomy of the nursing staff after the implementation of a standard replacement protocol. Todd et al. (2009), suggests that the use of electrolyte replacement protocols decrease delay in electrolyte replacement, increase effectiveness of electrolyte replacement, and eliminates variations in practice.

Similarly, in a retrospective study by Couture et al. (2013), post-surgical patients who were managed with an electrolyte replacement protocol maintained higher serum electrolyte concentrations than those treated with a traditional provider driven approach. 
In this study, the protocol group received more doses of electrolyte repletion than the control group, resulting in a higher percentage of daily electrolytes lab values in the desired range. Couture et al. (2013) reviewed 627 patient records and found that the use of an electrolyte replacement protocol led to improvement in serum electrolyte concentrations in a therapeutic range. In their study, Couture et al. (2013) noted that the protocol group compared to the control group received one or more doses of magnesium or phosphate, contributing to achievement of their target serum electrolyte levels. Additionally, there was a higher than desired level of potassium in the control group compared to the protocol group. Couture et al. (2013) established that the electrolyte repletion protocol used was safe and led to better control of serum potassium which translated to improved patient outcomes.

Pearson et al. (2018) reported that the use of standardized electrolyte replacement protocols for qualified patients can significantly decrease the time lag between the abnormal laboratory result and the administration of electrolyte replacement.

Additionally, the results of their study suggest that electrolyte replacement protocols also prevent episodes of arrythmias and other adverse events related to electrolyte imbalances including neurological and skeletal muscle dysfunction. In a retrospective study, Pearson et al. (2018) also discovered that improvement in the ease and timeliness of electrolyte replacement was indicated by a reduction of 4.4 minutes/patient/day in time spent on ordering, verifying, and administration of electrolyte replacement. Results also suggested decreased median time for replacement for all electrolytes from 146 minutes to 98 minutes (Pearson et al., 2018). The decrease in time was attributed to the decrease in the need to seek orders for electrolyte replacement and timeliness of administering the 
replacement doses. An added benefit to implementing a protocol included the timeliness and ease of using the replacement protocol and increased nurse satisfaction.

Likewise, in a retrospective observational study by Hammond et al. (2019), it was found that the development and institution of a patient specific algorithm for potassium replacement improved the effectiveness of potassium replacement. The study concluded that the target serum concentration of potassium was reached more often and was timelier with the implementation of a potassium replacement protocol. The researchers observed that there was no increase in daily laboratory testing with a standard replacement protocol compared to previous practice. The results suggested no evidence of unnecessary laboratory testing and no immediate adverse economic consequences. Additionally, in the absence of a standard potassium replacement protocol, patients achieved target serum levels in only $18 \%$ of patients, compared to $72 \%$ of the time after the implementation of a standard protocol (Hammond et al., 2019).

\section{Risks and Barriers to Electrolyte Replacement Protocols}

Multiple risks and barriers to the implementation of electrolyte replacement protocols exist on medical-surgical units including education, skills, and confidence levels of health care providers and registered nurses, as well as liability concerns. Zielenski et al. (2017), suggested that overtreatment of an electrolyte imbalance can unintentionally cause excess serum levels of that electrolyte and the potential for unintended side effects. In their research, the effectiveness and safety of a timed electronic assessment driven potassium replacement protocol (TARP) was compared with an electronic nurse-driven replacement protocol (NRP) (Zielenski et al., 2017). An electronic nurse-drive replacement protocol (NRP) requires the nurse to assess a patient's 
electrolyte status, replace the proper electrolyte and reassess the patient as needed. The NPR does not prompt laboratory testing at specific or consistent intervals, and the reliability and treatment relies heavily on the individual nurse's judgment. In this study, a total of 300 patients were treated for potassium replacement, with 152 patients in the TARP group and148 patients in the NRP group. The TARP group had a total of 564 instances of potassium replacement compared to 491 instances among the NRP group. The researchers found that of the 564 instances of replacement among the TARP group, the correct dose and reassessment was performed 416 times, or $73.8 \%$ of the time. However, of the 491 instances of potassium replacement in the NRP group, the correct dose and reassessment was only completed 117 times, or $23.8 \%$ of the time. The study concluded that the use of a TARP improved potassium replacement effectiveness and safety over the NRP without negatively affecting timeliness of care (Zielenski et al., 2017). The study also suggested that the implementation of the protocol increased nurses' confidence in identifying hypokalemia, and decision-making abilities in determining the appropriate electrolyte replacement dose and reassessment of the patient after electrolyte replacement.

In the absence of standardized protocols, replacement therapy leads to haphazard repletion of electrolytes without a valid indication, as well as economic consequences for inpatient facilities (Joseph et al., 2018). A retrospective chart review done by Joseph et al. (2018), reviewed provider behaviors with regards to ordering electrolyte replacement. Results indicated that there was significant variability in the practices of individual providers. The researchers also noted that some providers replaced electrolytes such as potassium, magnesium, and phosphate, despite higher serum levels. Joseph et al. (2018) 
indicated that the administration of electrolytes in the absence of a deficiency creates additional workload for providers and nurses and may cause adverse complications for patients with multiple comorbidities, such as cardiovascular and renal disease, resulting in longer hospital stays and poor outcomes.

\section{Nurses Knowledge}

Nurses are an essential part of the interdisciplinary team caring for the adult inpatient population. It is important for nurses to be aware of changes in the fluids and electrolyte status of their patients and be confident in the management, treatment, and care of these patients. The administration of electrolytes such as potassium, magnesium, and phosphorus can be critical to patient outcomes. Nurses must possess a baseline knowledge of the etiology of electrolyte disturbances and recognize potential complications related to deficiencies of these key electrolytes. To ensure the best possible outcomes for patients, nurses must be competent in interpreting labs values as well as in administering pharmacologic replacements of deficient electrolytes. For the implementation of electrolyte replacement protocols to be successful, nurses must be knowledgeable in dosing, route, time, and precautions of electrolyte administration. Günes \& Celik (2014) suggested that, nurses who were employed for ten years or more in acute inpatient nurse role had higher test score compared to nurses employed in the same role four years or less, related to knowledge of potassium administration. The knowledge gap in this study, although limited to one hospital, suggested that electrolyte protocols can be used to provide standards of practice and that more education should be provided to new nurses during the orientation and onboarding process. This study also indicates that additional education on electrolyte replacement and administration, for all nurses, 
can be used to improve knowledge about the care, management, and treatment of patients with electrolyte imbalances. A scarcity of literature exists related to nurses' knowledge of electrolyte replacement protocols in the inpatient setting, suggesting that this is an area requiring further study.

Next, the theoretical framework will be discussed. 


\section{Theoretical Framework}

The theoretical framework that was used to guide this research project was the Logic Model. The Logic Model, a theory of change, provided a methodical process of developing the education session used in this project (W.K. Kellogg Foundation, 2004). The Logic Model guided the development, implementation and evaluation of the educational intervention that was used. Each step in the Logic Model is crucial to developing and implementing the educational intervention, analyzing the data collected, and evaluating the outcomes. The Logic Model was applied to this clinical problem and was used in a step-by-step process to identify needs and create the desired outcomes with minimal unexpected outcomes (W.K. Kellogg Foundation, 2004). The steps of the Logic model include resources or input, activities, outputs, outcomes, and impact. Each step in the Logic Model is touched upon in the next paragraph, however will be explained in more detail under methodology.

\section{Figure 1}

The Logic Model. W. K. Kellogg Foundation, 2004

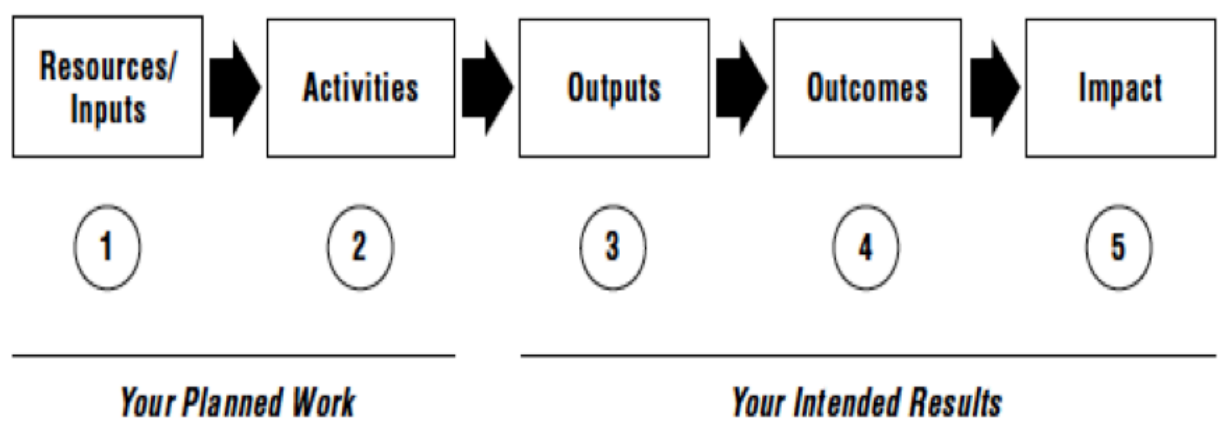

The first step in the Logic Model is planning. This step determines the resources or inputs available including identifying the site, sample, time limitations, equipment and project approval processes required to successfully develop the educational program. 
During the planning phase the resources were identified and the educational intervention and evaluation tool were designed. The educational intervention consisted of a presentation on electrolytes and replacement therapy protocols and the evaluation tool created consisted of a pre-posttest. It is important to determine what the educational intervention will consist of, what it will be evaluating, and how it will be evaluated. In this project, it will be nurse's knowledge of the management, treatment, and care of adult patients with electrolyte imbalances and will be evaluated using a pre-posttest format. Through the process of identifying the resources, it was important to identify barriers impacting the implementation of the program. The biggest barrier forecasted was IRB approval at the clinical site and time constraints of the nurses on the unit. The next step in the Logic Model is activities. The key activities included presenting the educational sessions and administering the pre-posttests. The output step of the model reflects measuring the impact of the educational intervention by analyzing the data from the preposttests. The desired goal was improvement in knowledge related to the management, treatment and care of patients with electrolyte imbalances.

The intended goals established in the of the activities step is the effect of the educational intervention on the participants. The goal of the outputs include improvement in knowledge of the care and management of patients with electrolyte imbalances. The next step in the process is outcomes, which include the intended benefits of successfully implementing the educational intervention. The intended outcomes reflect both shortand long-term benefits. The researcher should keep in mind that the planned activities may result in intended and unintended outcomes. The final step in the Logic Model is impact, or the benefits and changes that occur as a result of the process. If the research 
project is successfully implemented, the increased knowledge level of the participants can directly influence nursing practice and improve patient care and outcomes. The subsequent steps of the Logic Model, outputs, outcomes, and impact are evaluated once the educational intervention is implemented and data is collected and analyzed.

In the next section, the methodology for this research project will be discussed. 


\section{Method}

\section{Purpose}

The purpose of this research project was to determine if an educational program related to electrolyte replacement would improve nurse's knowledge related to standardized electrolyte replacement protocols and the management, treatment, and care of adult patients with electrolyte imbalances.

\section{Design}

The design for this research project included a pre-test and, an educational intervention followed by a post-test. The questionnaires were designed to evaluate nursing knowledge regarding electrolyte replacement protocols and the treatment of patients with electrolyte abnormalities.

Site

The site of this research project was the Providence Veterans Affairs Medical Center (PVAMC), a seventy-four-bed community hospital that services qualified veterans in Rhode Island and the surrounding areas. The specific unit used was $6 \mathrm{~B}$, a twentyseven-bed medical-surgical and telemetry unit.

\section{Sample}

A convenience sample of nurses who work on $6 \mathrm{~B}$ was used. Currently, 35 nurses work on this unit providing direct patient care. Participation in the educational intervention was completely voluntary. Inclusion criteria consisted of all full and part time registered nurses working on $6 \mathrm{~B}$ who provide direct patient care. Exclusion criteria included nurses from other units within the hospital, per-diem nurses, float pool nurses, travel nurses, and post baccalaureate registered nurse residents working on the unit. 
Exclusion criteria also included unit staff working on the unit outside of the registered nurse role.

\section{Procedure}

This research project was guided by the Logic Model. Steps in this model include input, activities, output, outcomes, and impact (W. K. Kellogg Foundation, 2004).

Input. This research project was submitted for approval to the PVAMC Institutional Review Board (IRB), as well as to the Rhode Island College (RIC) IRB and was determined to be an exempt level of review. Permission to complete the project was obtained from the medical surgical unit manager at the PVAMC. This individual assisted in arranging use of the unit conference room for all scheduled educational sessions. To ensure equal access, the educational program was offered to all registered nurses on the medical surgical unit who met the inclusion criteria. No vulnerable populations participated and no identifying demographic information was collected on the preposttests. Participation in the educational session was strictly voluntary.

Activities. The activities consisted of a pretest and an educational session, followed by a post-test. After IRB approval was obtained, an informational email was sent using the hospitals outlook email system to all registered nurses on the unit who met the inclusion criteria. The recruitment list was obtained from the Nurse Manager on 6B, and, with the manager's approval, the informational email was sent. The email outlined the purpose and process of the research project, the dates and times of the educational sessions, and that participation only required attendance at one of the scheduled educational sessions. The email also explained that participation was voluntary, how the data would be used, and that no identifying information would be collected. 
Additionally, an informational flyer detailing the purpose of the research project along with the dates and times of the educational sessions was posted on the unit. The educational sessions took place during the spring 2021 semester throughout the month of March, and all data was collected prior to and after each educational session. Data was analyzed in April 2021.

The pre-posttests assessed nursing knowledge related to the management, treatment, and care of adult patients with electrolyte imbalances using recommended electrolyte replacement protocols. The educational session was developed and included teaching points using a Power Point Presentation related to electrolyte replacement, the use of standardized electrolyte replacement protocols in the inpatient setting, and the benefits of implementing these types of replacement protocols for patients as well as staff. Sessions were offered on different days of the week, excluding the weekend, and were offered at different times and on day and evening shifts to allow for greater flexibility in attendance and to increase likelihood of participation by the nurses on the unit. Each nurse needed to attend only one session. A post-test was given at the end of each educational session.

The pre-posttest was developed by the researcher and was based on evidencebased research and best practices (NIH, 2019; Norris, 2019; Pearson et al., 2019). The pre-posttest consisted of ten questions and used multiple choice and true/false formatting. The same questions were used on both pre-posttests. No identifying information was solicited on the pre-posttests to ensure anonymity. Additional nurses attended the session; however, a section was included on the pre-posttest to indicate what unit the participant worked on and what role on the unit (RN, NA, LPN) they held. Only 
responses from registered nurses currently working on $6 \mathrm{~B}$ who met the inclusion criteria were used for analysis. The nurses received approximately ten minutes prior to the educational session to complete the pretest. The post-test used the same ten questions and was provided to each nurse immediately following the educational session. The participants were given 5-10 minutes to complete the post-test prior to leaving the conference room. As noted previously, the tests did not collect any identifying information, and the pre-posttests were not linked. The tests were saved for reference, locked securely in file box, and the answers to the test were not discussed after the sessions or posted.

Output. The output consists of the aggregate mean scores from the pre-test and post- tests. The outcome measures are the comparison between the means of the pre-test scores before the educational session and the mean scores of the post-test after the educational session. The pre-posttest scores were compared using simple descriptive statistics. The mean scores of pre-posttests were used to evaluate the effectiveness of the educational session and to determine if an increase in nurses' knowledge occurred following the educational intervention and to identify areas related to electrolyte replacement therapy that may need further education.

Impact. The desired impact of this research project was an increase in nursing knowledge evident by improved post-test scores regarding the management, care, and treatment of patients with electrolyte abnormalities. The collected data was presented to the Clinical Nurse Leader and the Unit Manager to determine any ongoing or additional educational needs or in-service education that would be beneficial to the nurses on $6 \mathrm{~B}$. 


\section{Measurement}

A ten-question pre-posttest was used to measure the effectiveness of an educational session by measuring an increase in nurses' knowledge of the management, care, and treatment of patients with electrolyte abnormalities. The questions used for the pre-posttest were developed using evidence-based literature and best practices, and PVAMC policies and procedures. The pre-posttests were reviewed and approved by the Unit Manager at the PVAMC, and approval was granted by both the PVAMC and RIC IRB.

\section{Challenges and Opportunities}

The PVAMC is supportive of staff participation in research and educational projects, as well as unit based and hospital wide research and quality improvement projects. The support of the unit manager provided an opportunity to impact nursing practice on the unit, as well as positively influence patient outcomes. In the process of implementing this research project, several challenges emerged. Challenges identified included lack of nurse participation in the educational sessions, time constraints needed for nurses to complete all the elements required of the project, acuity of the unit, and lack of available staff to cover the unit nurses to allow attendance. The pre-posttest was developed by the researcher for this project and therefore lacked testing for reliability. However, the confidence in the quality of the questions was increased by using best practice guidelines and drawing similar questions from a national test bank.

Opportunities for further education related to electrolyte management, treatment, and care of patients with electrolyte imbalances were identified. 


\section{Ethical Considerations}

Every effort was made to uphold ethical standards. This research project involved human subjects and was approved by both the PVAMC and RIC IRB. This research project was supported by the 6B unit manager and by the Unit Practice Council on 6B. The registered nurses that participated were made aware that involvement was strictly voluntary, and they were able to withdraw from the project at any time, for any reason, without penalty. No identifiable or demographic information of the participants were collected at any time during the research project.

\section{Analysis/Dissemination}

The results were evaluated using simple descriptive statistics. The pretest scores were collected and displayed in a bar graph to calculate trends as appropriate, and the same process was repeated for the post-test scores. The pre and post-test scores were then compared to identify any statistical significance of the educational intervention. The mean scores from the pre-posttests were compared to determine if there was an increase or improvement in knowledge after participating in an educational session. The data was also evaluated to determine which areas related to the management, treatment, and care of patients with electrolyte imbalances may need further education.

Additionally, the results suggest that a lack of knowledge may extend beyond the unit, potentially hospital-wide. The results were shared with the Unit Practice Council on 6B, the Unit Manager, and the Clinical Nurse Leader both who are responsible for addressing the educational needs of the staff on the unit. The results were displayed on the unit's bulletin board for the nurses to view. Displaying the poster board in the conference room allows the nurses to see the areas where knowledge was improved and 
areas that need further education and training. Given the positive feedback, this education may be considered for inclusion in new nurse orientations, as well as in-service opportunities for nurses on other units at the PVAMC.

Next, the results of the research project will be discussed. 


\section{Results}

A total of sixteen nurses attended the small group educational sessions. All of the nurses who participated in the educational session met the inclusion criteria. One nurse who met the inclusion criteria and attended the education session, completed the pre-test but was not able to complete the post-test. Fifteen of a possible 35 nurses completed the pre-test, educational session, and post-test $(\mathrm{n}=15 ; 42.9 \%)$.

The pre-test and post-test consisted of ten questions. The questions are available for review in Appendix D. Each question had a single correct response. Answers that were left unanswered or had multiple responses were counted as incorrect. The pre and post-test scores were aggregated and analyzed for improvement. Figure 2 represents a comparison of pre and post-test scores. The pretest and post-test mean scores were 9.2 and 12.6 respectively. Nine out

\section{Figure 2}

\section{Comparing Pre-Test and Post-Test Scores}

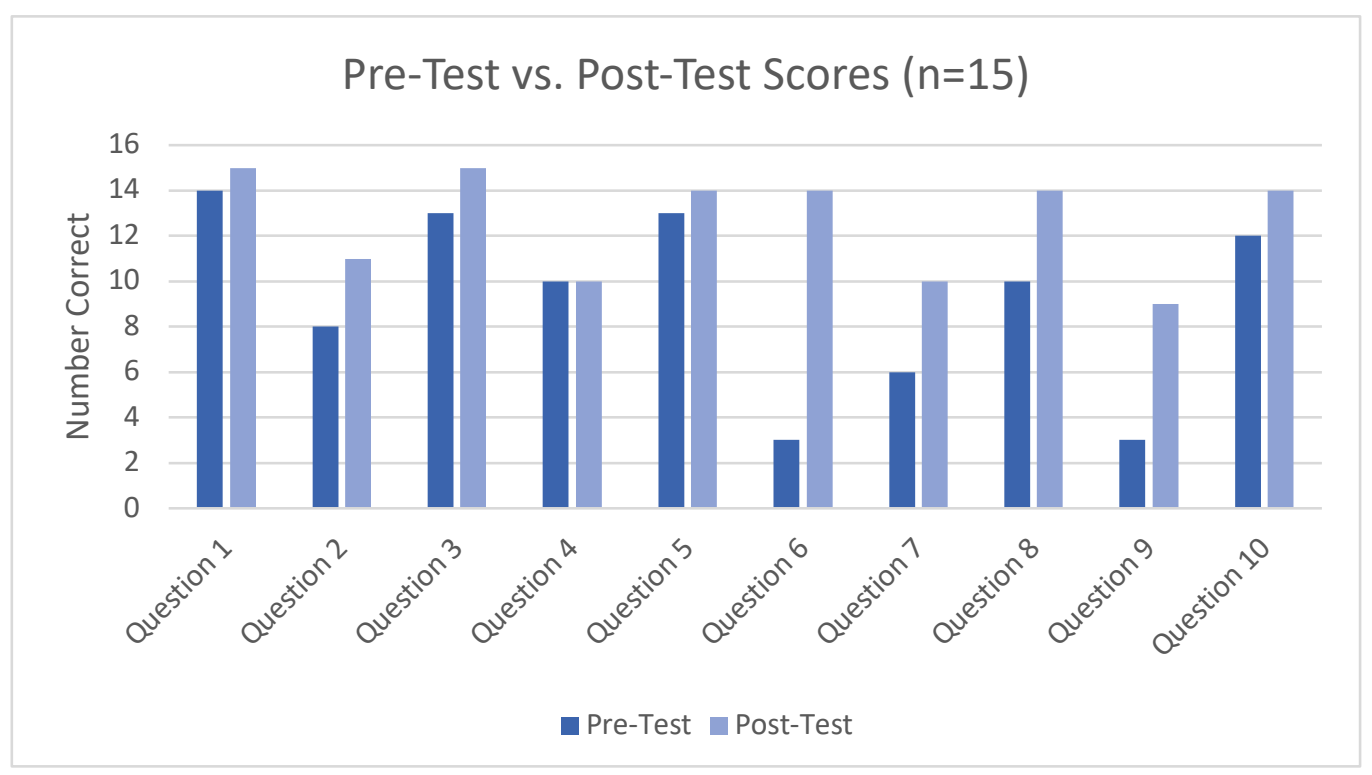


of the ten questions showed improvement after the small group educational sessions. The ten questions on the pre-posttest were comprised of five topics which included the role of electrolytes in the body, clinical manifestations of electrolyte deficiencies, relationships of electrolytes in the body, considerations and precautions for electrolyte replacement and recommendations for electrolyte repletion.

The pre-test and post-test mean percentages are presented in Figure 3.

\section{Figure 3}

\section{Mean Percentages}

\begin{tabular}{|c|c|c|}
\hline Question & Pre-Test & Post-Test \\
\hline 1 & $93.3 \%$ & $100 \%$ \\
\hline 2 & $53.3 \%$ & $73.3 \%$ \\
\hline 3 & $86.7 \%$ & $100 \%$ \\
\hline 4 & $66.7 \%$ & $66.7 \%$ \\
\hline 5 & $86.7 \%$ & $93.3 \%$ \\
\hline 6 & $20 \%$ & $93.3 \%$ \\
\hline 7 & $40 \%$ & $66.7 \%$ \\
\hline 8 & $66.7 \%$ & $93.3 \%$ \\
\hline 9 & $20 \%$ & $60 \%$ \\
\hline 10 & $80 \%$ & $93.3 \%$ \\
\hline Pretest and Posttest & $61.3 \%$ & $84 \%$ \\
Mean Percentages & & \\
\hline
\end{tabular}

The pre-test scores and post-test scores showed a $22.7 \%$ increase in overall correct answers from pre-test $(61.3 \%)$ to post-test scores $(84 \%)$. The results indicated the greatest overall improvement in knowledge after the educational session was approximately equal between the topics of recommendations for electrolyte repletion (26.7\%), and electrolyte replacement considerations and precautions $(26.6 \%)$. The individual questions that indicated the greatest increase in knowledge after the educational session were question six $(+73.3 \%)$ and question nine $(+40 \%)$. 
The pre-posttest had one question regarding the role potassium plays in the body, question one. The majority of the participants did well on both the pre and post-tests, with a slight improvement of $6.7 \%$ after the educational intervention for question one. The topic of relationships between electrolytes in the body was reflected in question two, which asked if there was a correlation between potassium and magnesium. Question two had overall improvement of $20 \%$ from the pre-test compared to the post-test. Question three asked about the clinical manifestations of a magnesium deficiency. The pretest indicated a score of $86.7 \%$, and a post-test score of $100 \%(+13.3 \%)$ for question three. Question three had the least improvement, however the small percentage of improvement is due to the majority of participants answering correctly on both the pre and post-tests.

There were four questions on the pre-posttest that covered the recommendations for electrolyte repletion $(4,5,6$, and 7$)$, which resulted in a $26.7 \%$ increase in knowledge in this topic area following the educational session. Question four regarding the recommendations for magnesium replacement remained unchanged before and after the educational session at $66.7 \%$. Question five regarding phosphorus replacement had the least percentage of improvement at $6.6 \%$, however the majority of participants answering correctly on both the pre and post-tests. Question six, which asked about the recommended dose of oral potassium replacement for a patient with a serum lab value of $3.3 \mathrm{mEq} / \mathrm{L}$ showed the most significant improvement from pre-test to post-test. Question six had a pre-test score of $20 \%$ and a post-test score of $93.3 \%$, reflecting an increase of 73.3\% after the educational session. Question seven was a question regarding the correct infusion time for intravenous phosphorus replacement showed the second greatest improvement among the topic area of considerations for electrolyte replacement. The 
findings for question seven reflected an improvement from $40 \%$ on the pre-test to $66.7 \%$ on the post-test $(+26.7 \%)$.

There were three questions on the pre-posttest that covered the topic area of considerations and precautions for electrolyte replacement $(8,9$, and 10$)$, which an overall knowledge improvement of $26.6 \%$ after the educational session. Question eight refers to the maximum infusion rate for intravenous potassium and resulted in pre-test score of $66.7 \%$ and a post-test score of $93.3 \%(+26.6 \%)$. Question nine which asked the sequence of administration for intravenous magnesium and potassium for a patient with a concomitant deficiency reflected the most improvement of this topic area after the educational session. Question nine had a pre-test score result of $20 \%$ compared to the post-test score of $60 \%$, an improvement of $40 \%$ after the implementation of the educational session. Question ten asked about considerations for specific patient populations when repleting electrolytes, and resulted in an improvement of $13.3 \%$ between the pre-test and post-test.

Improvement in all five topic areas demonstrated that the implementation of an electrolyte replacement educational program was effective in improving the medicalsurgical nurse's knowledge of management, treatment, and care of adult patients with electrolyte abnormalities.

Next, the summary and conclusions will be discussed. 


\section{Summary and Conclusion}

The incidence of electrolyte imbalances in the acute inpatient population is a common problem related to multiple comorbidities, medication use, side-effects, and interactions and other treatments. Electrolyte imbalances have the potential to increase patient morbidity and mortality rates. The purpose of this research project was to determine whether the implementation of an educational intervention would improve the nurses' knowledge regarding management, treatment, and care of adult patients with electrolyte abnormalities.

A search for studies meeting the inclusion criteria was completed using Ebscohost databases CINAHL, PubMed, and MEDLINE. A literature review which discussed the basic of electrolytes, electrolyte imbalances, management of electrolyte replacement, replacement protocol, benefits and risks of replacement protocol, and nurse's knowledge was presented. The theoretical framework outlined the steps of The Logic Model, a change theory, in the development, implementation, and evaluation of the educational intervention.

According to Wolf and Hilewitz (2014), the prevalence of hypomagnesaemia in the general inpatient population is over $20 \%$, and can be associated with increased mortality. Hypokalemia can also be found in over $20 \%$ of patients during their hospital stay (Zielenski et al., 2017). Deficiencies in these electrolytes are common among patients on a medical-surgical unit. It is important that nurses providing care for the inpatient population are able to identify patients who may be at risk for electrolyte imbalances to prevent complications and prolonged hospital admissions. Additionally, nurses can make medications errors and increase patient complications if they have 
insufficient knowledge and lack best practices when administering electrolyte replacement.

Todd et al. (2009) suggests that the use of electrolyte replacement protocols decreases a delay in electrolyte replacement, increases effectiveness of replacement therapy, and eliminates variations in practice. Pearson et al. (2019) demonstrated that the use of standardized electrolyte replacement protocols for qualified patients can significantly decrease the time lag between the abnormal laboratory result and the administration of electrolyte replacement. Additionally, the implementation of nurse driven electrolyte replacement protocols was shown to improve efficacy and timeliness of electrolyte replacement and improved nurse autonomy and provider satisfaction.

This research project took place at the PVAMC on 6B, a medical-surgical unit. In order to support the implementation of a nurse-driven electrolyte replacement protocol on the unit, baseline knowledge of electrolyte monitoring and replacement therapy was assessed and education provided. The educational intervention was developed using basic knowledge regarding the monitoring and replacement of electrolytes including potassium, magnesium, and phosphorus. The educational intervention effectiveness was measured using a pre-posttest method. The questions on the pre-posttests included topics such as electrolyte function and relevance in the body, serum lab values, and specific electrolyte replacement recommendations. Fifteen of a possible 35 nurses completed the pre-test, educational session, and post-test. Scores increased from a mean percentage pre-test score of $61.3 \%$ to a post-test mean percentage score of $84 \%$, demonstrating an improvement of $22.7 \%$. Nine out of the ten questions showed improvement on the posttest. 
Similar to Gunes and Celik's (2014) study, the pre-test results of this project indicate that nurses have insufficient knowledge regarding intravenous potassium and proper administration of potassium. Recommendations from Gunes and Celik (2104) indicated that nursing education programs and in-service training need to be developed to address this knowledge gap. Aslam et al. (2017) work supports the findings of this project suggesting that educational interventions regarding fluid and electrolyte balance and monitoring should be implemented and that educational strategies have the potential to increase knowledge and skills, as well as clinical judgment of nurses regarding electrolyte administration.

Nurses must possess a baseline knowledge of the etiology of electrolyte disturbances and recognize potential and possible complications related to deficiencies of these key electrolytes. To ensure the best possible outcomes for patients, nurses must be competent in interpreting labs values as well as in administering pharmacologic replacements of deficient electrolytes. For the implementation of electrolyte replacement protocols to be successful, nurses must be knowledgeable in dosing, route, time. and precautions of electrolyte administration.

\section{Limitations}

Several limitations were identified in this project. Implementation of the educational session were scheduled on several days and on different shifts and times to accommodate the majority of the nurses on the unit. Due to multiple variables, such as staffing levels and patient acuity, many nurses were still not able to attend, generating a small sample size. Additionally, a research study that concurrently running overlapped with the educational sessions planned. Other limitations included the nurses' time 
constraints, staff call outs, staff floated off unit to cover the COVID unit, lack of interest, and lack of commitment to complete the pre-posttest and educational session.

Additionally, interruptions during the educational session required nurses to leave in order to care for patients, answer phone calls, or attend to necessary tasks. The preposttest was developed by the researcher, thus limiting reliability.

Next, recommendations and implications for advanced nursing practice will be discussed. 


\section{Recommendations and Implications for Advanced Nursing Practice}

Nurses are an essential part of the interdisciplinary team caring for the adult inpatient population. Nurses at the bedside play an important role in the effective management, treatment, and care of adult patients with electrolyte abnormalities. Nurses' comprehensive knowledge of monitoring and repletion of electrolytes such as potassium, magnesium, and phosphorus can play a significant role in decreasing hospital length of stay and reducing complications related to electrolyte abnormalities. It is important for nurses to be aware of changes in the fluids and electrolyte status of their patients and be confident in the management, treatment, and care of these patients.

The APRN has the skills to identify gaps in current practices and collaborate with nursing leadership to impact change. The APRN, as has been demonstrated with this research project, collaborated with the unit manager in identifying a knowledge gap among unit nurses. The opportunity then exists for APRNs to take the initiative in creating educational programs that address these gaps in knowledge and skills. The gaps identified in this project included analyzing and interpreting electrolyte laboratory values, notifying the provider that electrolyte repletion may be required, and understanding considerations and precautions of administering electrolyte replacement.

Currently, within the PVAMC no standardized practices or policies exist regarding the repletion of electrolytes, and the decision is left to individual providers. Informed by research, the APRN has the skill sets to facilitate the development and implementation of a standardized evidence-based electrolyte replacement protocol. Development and implementation will require the APRN to engage in interdisciplinary collaboration. The APRN can use a variety of settings within the hospital environment 
such as new nurse orientation and unit-based in-services to educate nurses. Additionally, the APRN may utilize a variety of teaching strategies such as one-on-one training, skill labs, or classroom settings to provide education on the management of electrolyte abnormalities. In this project, education was focused on the nurses on $6 \mathrm{~B}$, a medicalsurgical unit, and education was performed in a group format.

With the successful implementation of a standardized evidence-based electrolyte replacement protocol on $6 \mathrm{~B}$, the protocol, under the direction of the APRN, could be phased in on other nursing units, potentially producing increased positive patient outcomes. Implementing the protocol hospital-wide will require leadership and collaboration with nursing administration, nurse educators, clinical nurse specialists, unit nurse managers, nurses, and other health care providers and would demonstrate the APRN's commitment to best practices.

Currently, the nursing staff on the unit often defaults to the provider to identify electrolyte deficiencies and decide adequate replacement doses for the patient creating inconsistent and varying practices from one health care provider to another. With the PVAMC being a teaching hospital, the APRN has the opportunity to also educate rotating interns and residents to provide consistency among health care providers by introducing them to and educating on the benefits of a standardized electrolyte replacement protocol.

The purpose of this research project was to determine whether the implementation of an educational intervention would improve the medical-surgical nurses' knowledge of management, treatment, and care of adult patients with electrolyte abnormalities. Moving forward, a goal is to develop nurse driven electrolyte replacement protocols. As with any change, it is important for the APRN to evaluate the effectiveness of the standardized 
replacement protocols before phasing in a nurse-driven protocol. The APRN recognizes that research will be ongoing to ensure a nurse driven electrolyte replacement protocol reflects best practices that promote safety and efficacy in providing electrolyte repletion therapy and reduces complications of electrolyte deficiencies.

The PVAMC supports and encourages nurse driven research and quality improvement projects as a mechanism to improve patient care and inform current policies and procedures. As part of the dissemination plan, this writer reviewed the results of the pre-posttest with the 6B Nurse Manager and the Clinical Nurse Leader on the unit. Areas in which nurses needed additional education were shared and ideas for developing and implementing a standardized electrolyte replacement protocol on the unit were discussed. Plans are underway to share the result of this study with the Unit Practice Council and to request permission to provide additional unit-based education based on the results of the post-test and nurse participant's feedback following the educational intervention. Initial conversation has been initiated with other APRNs and Nurse Managers within the PVAMC to offer the educational intervention from this quality improvement project to other inpatient units, outpatient clinics, Hospital in Home, and home-based primary care service areas, thus promoting a consistent hospital-wide protocol for electrolyte management and treatment. Ultimately, if successful at the PVAMC, this research project has the potential of being disseminated to other Veterans Administration Medical Centers in the region and nationally. With this opportunity, APRNs in this health care system, can facilitate the implementation of a standardized or nurse-driven electrolyte protocol that can have far-reaching impact on the quality of nursing care and patient outcomes. 


\section{References}

Aslam S., Afzal M., Kousar R., Waqas A., \& Gilani S. (2017). The assessment of nurses' knowledge and practices about fluid and electrolytes monitoring and administration among cardiac surgery patients: A case of Punjab Institute of Cardiology, Int. J. Appl. Sci. Biotechnol. Vol 5(2), 208-215.

Ayuk, J., \& Gittoes, N. J. L. (2011). How should hypomagnesaemia be investigated and treated? Clinical Endocrinology, 75(6), 743-746. https://doiorg.ric.idm.oclc.org/10.1111/j.1365-2265.2011.04092.x

Couture, J., Létourneau, A., Dubuc, A., \& Williamson, D. (2013). Evaluation of an electrolyte repletion protocol for cardiac surgery intensive care patients. The Canadian Journal of Hospital Pharmacy, 66(2), 96-103.

Ghany, M. (2019). Developing nursing standards for maintaining fluid and electrolyte balance for critically ill patients in intensive care units. Journal of Intensive and Critical Care, 5(1:4), 1-7.

Gooch, M. D. (2015). Identifying acid-base and electrolyte imbalances. The Nurse Practitioner, 40(8), 37-42. doi: 10.1097/01.NPR.0000469255.98119.82.

Güneş, A., \& Senol Celik, S. (2014). Knowledge and practices of nurses concerning intravenous potassium administration in a Turkish hospital. Australian Journal of Advanced Nursing, 32(1), 16-22.

Hammond, D. A., King, J., Kathe, N., Erbach, K., Stojakovic, J., Tran, J., \& Clem, O. A. (2019). Effectiveness and safety of potassium replacement in critically ill patients: A retrospective cohort Study. Critical Care Nurse 39, (1), e13-e18. http://doiorg.ric.idm.oclc.org/10.4037/ccn2019705 
Harrington, L. (2005). Potassium protocols: in search of evidence. Clinical Nurse Specialist CNS, 19(3), 137-141.

Hemstreet BA, Stolpman N, Badesch DB, May SK, \& McCollum M. (2006). Potassium and phosphorus repletion in hospitalized patients: implications for clinical practice and the potential use of healthcare information technology to improve prescribing and patient safety. Current Medical Research \& Opinion, 22(12), 2449-2455.

Hoekstra, M., Vogelzang, M., Drost, J.T. et al. (2010). BMC Med Inform Decis Mak. https://doi.org/10.1186/1472-6947-10-5

Jensen, H. K., Brabrand, M., Vinholt, P. J., Hallas, J., \& Lassen, A. T. (2015). Hypokalemia in acute medical patients: risk factors and prognosis. American Journal of Medicine, 128(1), 60-67.e1. https://doiorg.ric.idm.oclc.org/10.1016/j.amjmed.2014.07.022

Joseph, T.T., DiMeglio, M., Huffenberger, A. et al. (2018). Behavioral patterns of electrolyte repletion in intensive care units: lessons from a large electronic dataset. Sci Rep 8, 11915. https://doi.org/10.1038/s41598-018-30444-3

Kardalas, E., Paschou, S. A., Anagnostis, P., Muscogiuri, G., Siasos, G., \& Vryonidou, A. (2018). Hypokalemia: a clinical update. Endocrine Connections, 7(4), R135R146. https://doi-org.ric.idm.oclc.org/10.1530/EC-18-0109

Kear, T. M. (2017). Fluid and electrolyte management across the age continuum. Nephrology Nursing Journal: Journal of The American Nephrology Nurses' Association, 44(6), 491-496. 
Leilah MAG, Kandeel NA, Shebl AM, \& Mansour HE. (2019) Developing nursing standards for maintaining fluid and electrolyte balance for critically ill patients in intensive care units. J Intensive \& Critical Care, 5(1), 4.

Norris, T. L. (2019). Porth's pathophysiology: Concepts of altered health states. (10th ed.). Philadelphia, PA: Wolters Kluwer Health/Lippincott Williams \& Wilkins.

Owen, P., Monahan, M. F., \& MacLaren, R. (2008). Implementing and assessing an evidence-based electrolyte dosing order form in the medical ICU. Intensive \& Critical Care Nursing, 24(1), 8-19.

Pearson, D. J., Sharma, A., Lospinoso, J. A., Morris, M. J., \& McCann, E. T. (2018). Effects of electrolyte replacement protocol implementation in a medical intensive care unit. Journal of Intensive Care Medicine (Sage Publications Inc.), 33(10), 574-581. https://doi-org.ric.idm.oclc.org/10.1177/0885066616679593

Scotto, C. J., Fridline, M., Menhart, C. J., \& Klions, H. A. (2014). Preventing hypokalemia in critically ill patients. American Journal of Critical Care, 23(2), 145-149. https://doi-org.ric.idm.oclc.org/10.4037/ajcc2014946

Tazmini, K., Nymo, S. H., Louch, W. E., Ranhoff, A. H., \& Oie, E. (2019). Electrolyte imbalances in an unselected population in an emergency department: A retrospective cohort study. PLOS ONE, 14(4), e0215673. https://doi.org/10.1371/journal.pone.0215673

Todd S.R., Sucher J.F., Moore L.J., Turner K.L., Hall J.B., \& Moore F.A. (2009). A multidisciplinary protocol improves electrolyte replacement and its effectiveness. American Journal of Surgery, 198(6), 911-915. https://doiorg.ric.idm.oclc.org/10.1016/j.amjsurg.2009.04.032 
W. K. Kellogg Foundation. (2004). W.K. Kellogg Foundation Logic Model development guide. Retrieved from: https://www.wkkf.org/resourcedirectory/ resource/2006/02/wk-kellogg-foundation-logic-model-developmentguide.

Wolf, F., \& Hilewitz, A. (2014). Hypomagnesaemia in patients hospitalized in internal medicine is associated with increased mortality. International Journal of Clinical Practice, 68(1), 111-116. https://doi-org.ric.idm.oclc.org/10.1111/ijcp.12260

Zielenski, C., Crabtree, A., Le, T., Marlatt, A., Ng, D., \& Tran, A. (2017). Implementation of a timed, electronic, assessment-driven potassium-replacement protocol. American Journal of Health-System Pharmacy: AJHP: Official Journal of The American Society of Health-System Pharmacists, 74(12), 927-931. https://doi-org.ric.idm.oclc.org/10.2146/ajhp160378 


\section{Appendix A}

\section{Informational Email}

Dear 6B Registered Nurses,

My name is Sarah-Jane Santos and I am a graduate student at Rhode Island College, and a registered nurse on 6B here at the Providence VA Medical Center. The program requires that I complete a master's research project, and I have developed a research project for the medical-surgical registered nurses on $6 \mathrm{~B}$.

You are being asked to participate in this research project titled Knowledge of Electrolyte Replacement Protocols Among Medical-Surgical Registered Nurses: A Research Project. The purpose of this project is to develop an evidence-based educational program for nurses regarding the management, treatment and care of adult patients with electrolyte abnormalities.

All full time and part-time nurses on 6B are invited to participate. Participation is completely voluntary, and you can withdraw at any time. If you choose to participate, you will be asked to attend a 20 -minute educational session and complete a 10 -question multiple choice pre and posttest which should take approximately 5 minutes each to complete. The total time for completion of this educational activity will be approximately 30 minutes. There will be no identifying information collected during the process of the research project. Data will be collected from the pre and posttests and at the conclusion of the research project the data will be compared to identify any improvement in knowledge. 
If you have any questions regarding this research project, please contact me at Sarah-Jane.Santos@va.gov or 401-273-7100 Ext 13587. Thank you for taking the time to read this email and for your potential participation in this research project.

Sincerely,

Sarah-Jane Santos, BSN, RN-BC

Sarah-Jane.Santos@va.gov

Rhode Island College 


\section{Appendix B}

\section{Informational Flyer}

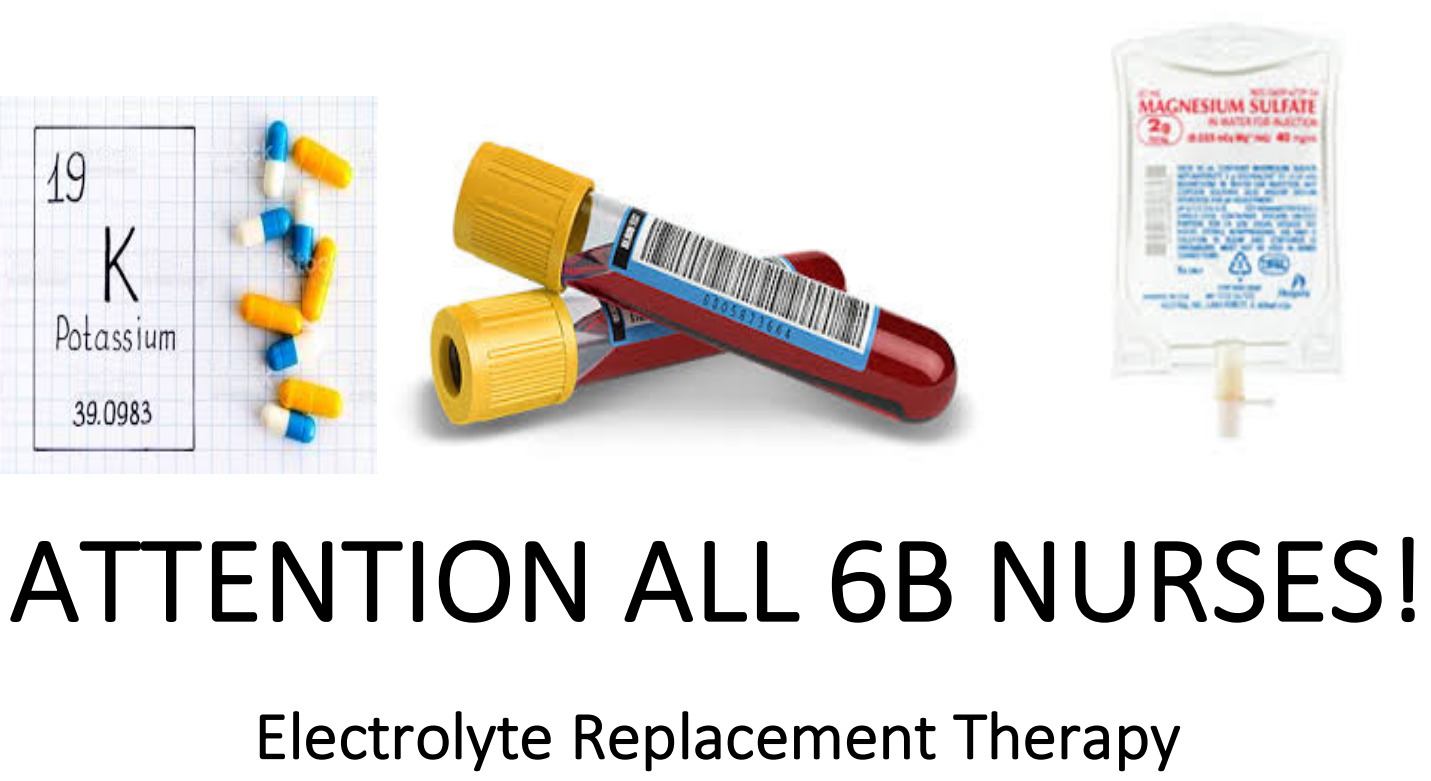

You are invited to participate in an education program to improve your knowledge about the management, treatment and care of adult patients with electrolyte abnormalities.

\begin{tabular}{|c|c|}
\hline Dates & Times \\
\hline March 2, 2021 & $12: 00 \mathrm{pm} \mathrm{\&} \mathrm{12:30} \mathrm{pm}$ \\
\hline March 4, 2021 & 7:00pm \& \& 7:30 pm \\
\hline March 8, 2021 & 6:00am \& 6:30am \\
\hline March 12, 2021 & $12: 00 \mathrm{pm} \mathrm{\&} \mathrm{12:30pm}$ \\
\hline
\end{tabular}

Each session will take approximately 30 minutes to complete and consists of a pre-test, educational program and post-test.

Sessions will be held in the 6B conference room.

For additional information please contact:

Sarah-Jane Santos, BSN, RN-BC

Sarah-Jane.Santos@va.gov 
Appendix C

Educational Intervention Power Point

Knowledge of Bectrolyte Replacement Protocols Among Medical-Surgical Nurses

A RESEARCH PROJECT

SARAH-JANE SANTOS, BSN, RN-BC

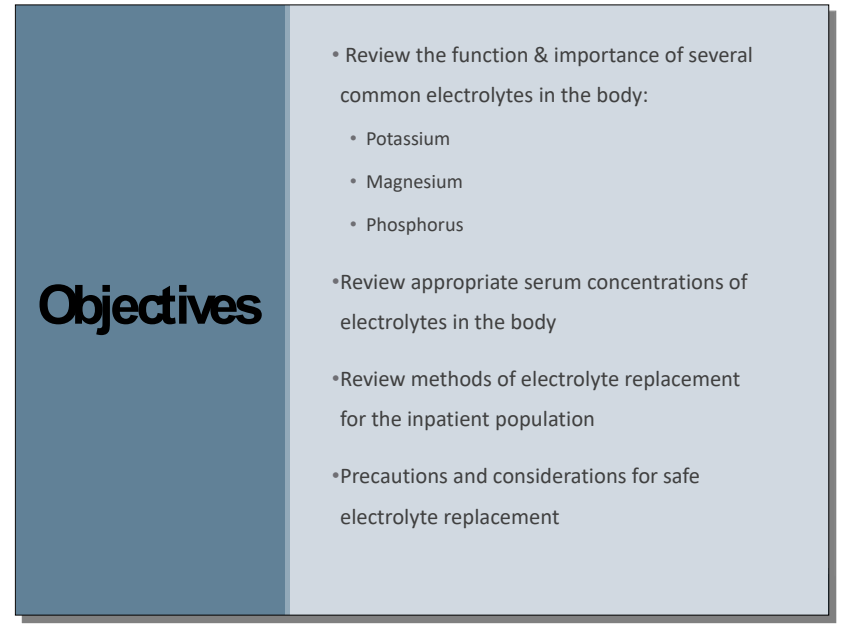

\section{Bectrolyte Overview}

Electrolytes are considered to have four main functions within the body:

- Cell metabolism and contribution to body structures

- Facilitation of fluid movement between different body compartments

- Maintenance of acid-base balance

- Maintenance and production of membrane potentials in the nerve and muscular cells 


\section{Bectrolyte Imbalances}

- Electrolyte abnormalities lead to increased emergency room visits,

hospital readmission rates and is associated with an increased 30-day

and one-year mortality rate (Tazmini, 2019)

-Imbalances of electrolytes can cause additional complications

among hospitalized patients

-Electrolyte levels must be corrected for the body to maintain acid base balance and continue normal bodily functions (NIH, 2019)

\section{Potassium}

- Potassium is the second most prevalent intracellular cation and the most abundant cation within the intracellular fluid

-Potassium in the extracellular fluid is significantly less, with normal levels measuring between $3.5-5 \mathrm{mEq} / \mathrm{L}$ Potassium is absorbed by the body in the small intestines

- Excretion is controlled by the kidneys with a majority being excreted in urine, the remaining excreted in stool and sweat

(Norris, 2019)

\section{Potassium: Function \& Importance}

- Potassium plays a key role in maintaining normal cell function using a pump called the Na+-K+-ATPase pump (Kardalas et al., 2018)

Constant movement of potassium across the cell membranes creates an action potential which many tissues rely on to function properly (Norris, 2019)

- Nerve conduction of cardiac, skeletal and smooth muscle

- Carbohydrate, glucose and amino acid metabolism

- Assists in the body's acid-base balance 

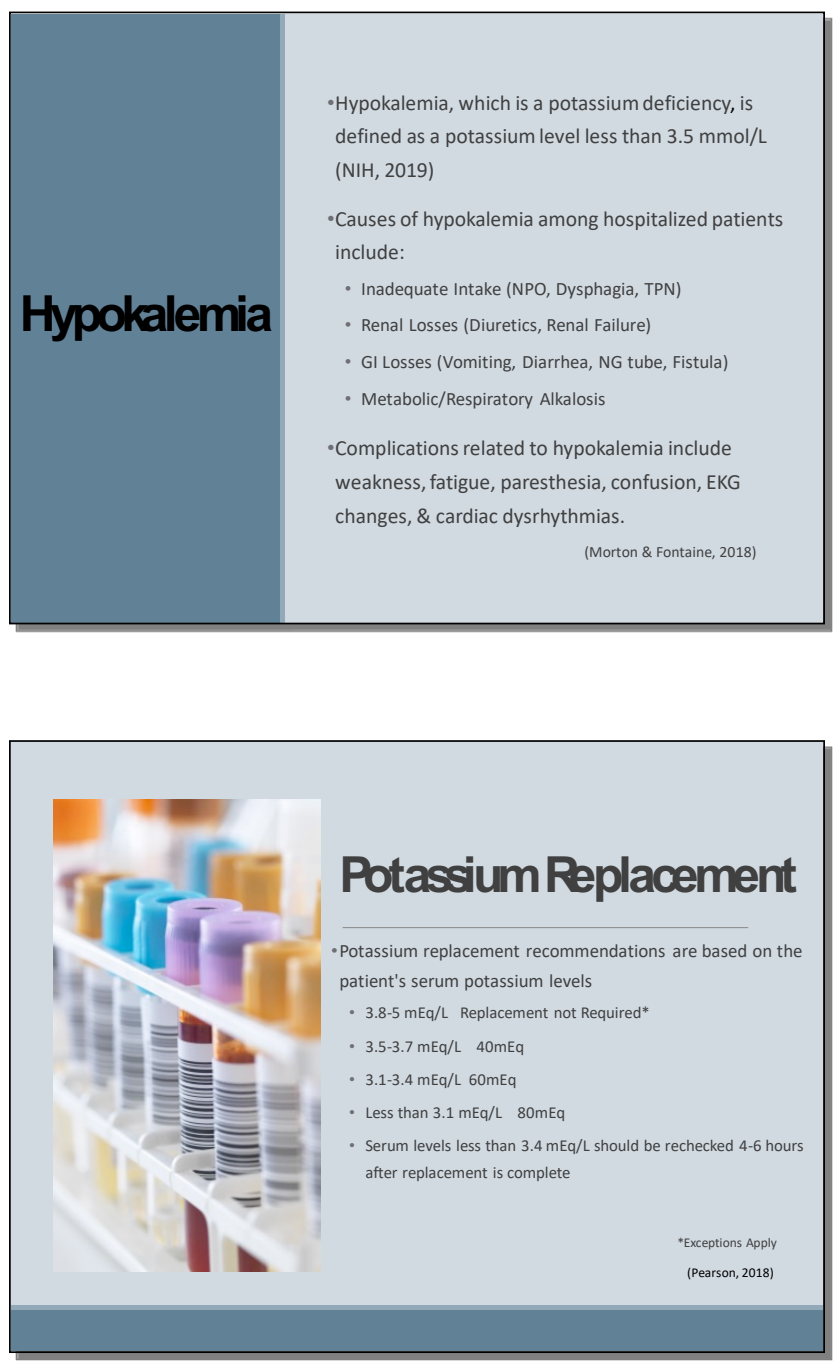

\section{Considerations}

Considerations when administering potassium replacement may include:

- If patient can tolerate, oral administration is preferred

- Serum levels less than $3.1 \mathrm{mEq} / \mathrm{L}$ consider intravenous

- Serum potassium levels should be rechecked as needed

- Consider patient age, weight, urine output, BUN/Cr levels

- General Rule: Each $10 \mathrm{mEq}$ of KCL administered is expected to raise serum K+ level by $0.10 \mathrm{mEq}$ (Ex: $40 \mathrm{mEq}$ administered $=$ increase of 0.40 ) 


\section{Precautions}

Precautions for potassium replacement may include:

- Repeat EKGs and consideration of continuous cardiac monitoring

- Maximum infusion rates:

- Peripheral Line $10 \mathrm{mEg} / \mathrm{hour}$

- Central Line $20 \mathrm{mEq} / \mathrm{hour}$

- Digoxin Levels

- Concomitant Hypomagnesemia

- Use caution in patients with ESRD, ARF or Renal Insufficiency, DKA, Urethral Obstruction

\section{Magnesium}

-Magnesium is an intercellular cation, second only to potassium within the intracellular fluid (Wolf \& Hilewitz, 2014)

- Normal magnesium concentrations in adults range from 1.3-2.1 mEq/L (Norris, 2019)

- Magnesium is absorbed in the intestines (jejunum), however only 25-65\% of magnesium in our diet is absorbed (Norris, 2019)

- Regulation of magnesium reabsorption and excretion is controlled by the kidneys, with excretion in the urine (Norris, 2019)

\section{Magnesium: Function \& Importance}

-Magnesium plays an important role in protein synthesis and carbohydrate metabolism

-Intracellular enzyme reactions, ADP --> ATP

-Active transport of calcium and potassium across cell membranes

- Nerve impulse conduction

- Muscle contraction

- Maintenance of normal cardiac rhythms 


\section{Hypomagnesemia}

-Hypomagnesemia is defined at a serum concentration of less than $1.8 \mathrm{mg} / \mathrm{dL}(\mathrm{NIH}, 2019)$

- Causes of low magnesium in hospitalized patients include (Wolf \& Hilewitz, 2014):

- Impaired PO intake (ETOH, malnutrition, Small Bowel Surgery)

- Treatment with Diuretics (Loop \& Thiazide), Antibiotics (Aminoglycosides)

- Diabetic Ketoacidosis, DKA

- Hyperparathyroidism, Hyperaldosteronism

- Complications related to hypomagnesemia can include personality changes, tetany muscle weakness, fatigue, numbness, nystagmus, hypertension, tachycardia, ventricular arrythmias (Wolf \& Hilewitz, 2014)

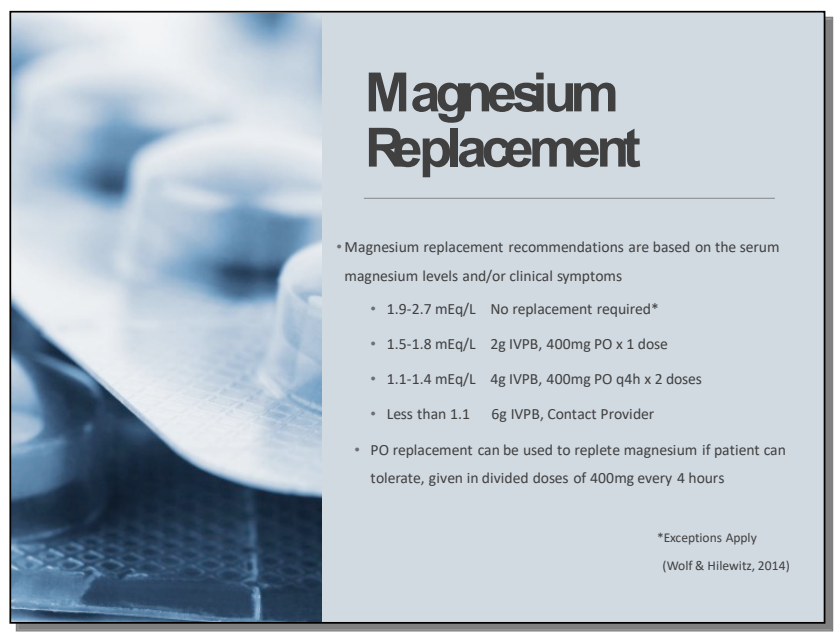

\section{Considerations \& Precautions}

Considerations when administering magnesium replacement may include:

- Oral vs. Intravenous route determined based on severity of magnesium deficiency

- Serum magnesium levels should be checked four hours after repletion if levels were $<1.1 \mathrm{mEq} / \mathrm{L}$

- Symptomatic hypomagnesemia should be treated with IV replacement

- Low serum potassium and albumin are both common with hypomagnesemia

- If necessary, administer intravenous magnesium replacement followed by intravenous potassium replacement

Precautions may include:

- Peripheral IV access, infuse at rate of $1 \mathrm{~g} /$ hour

- Serum levels $<1$, notify provider and complete neurological checks

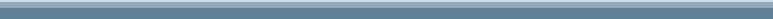




\section{Phosphorus}

- Phosphorus is an intracellular anion found mostly in the bones and teeth with a small

amount found in the soft tissues and extracellular fluid (Kear, 2017)

- Normal level of phosphorus within the adult human body is $2.5-4.5 \mathrm{mg} / \mathrm{dL}$ ( NIH, 2019)

- Phosphorus is absorbed in the intestines, absorption of phosphorus can be negatively affected by simultaneous ingestion of other substances such as calcium magnesium and aluminum (Norris, 2019)

- The kidneys, bones and intestines regulate phosphate levels, excreting excess phosphate though urine based on the total concentration within the body (Norris, 2019)

\section{Phosphorus: Function \& Importanœ}

-Phosphorus is a structural component of bones and teeth

- Phosphorus plays a key role in many bodily functions including:

- Energy production

- Gene transcription

- Enzyme activation

- Maintenance of acid-base balance

(Wolf \& Hilewitz, 2014)

\section{Hypophosphatemia}

-The normal level of phosphorus within the adult human body is $2.5-4.5 \mathrm{mg} / \mathrm{dL}$ (NIH, 2019)

-Causes of low serum phosphate levels may include (Morton \& Fontaine, 2018):

- Decreased absorption (Diarrhea, Vitamin D Deficiency, ETOH, Malnutrition)

- Medications (Antacids, Laxatives)

- DKA, Hyperparathyroidism

- Renal Tubular Defects

- Complications of low phosphate in the body include tremor, ataxia, confusion, seizures, anemia, bone pain, muscle weakness (Morton \& Fontaine, 2018) 


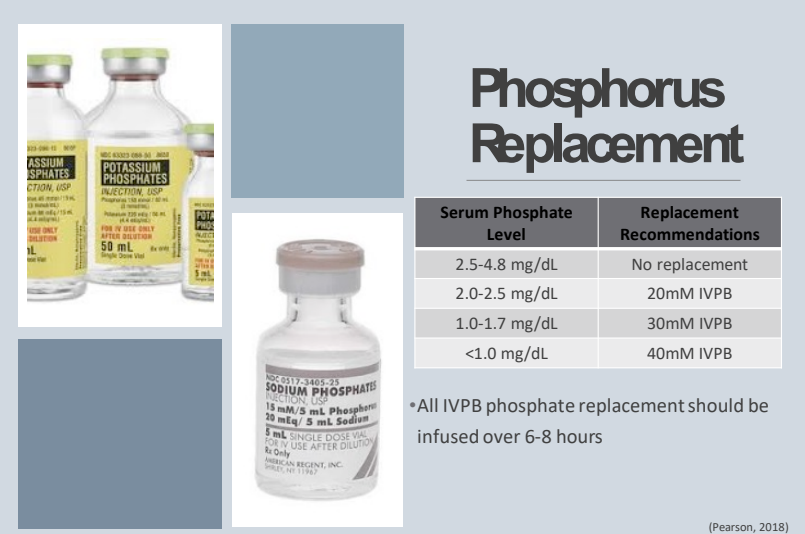

(Pearson, 2018)

\section{Considerations \& Precautions}

Considerations when administering phosphate replacement may include (Pearson, 2018):

- Monitor $\mathrm{K}+$ and $\mathrm{Na}+$ levels, levels are impacted by replacement with Potassium Phosphate vs.

Sodium Phosphate

- Repeat serum phosphate values as needed

- Infuse phosphate replacement slowly, typically over eight hours

Precautions related to phosphate replacement include (Pearson, 2018):

- Use cautiously in patients with $\mathrm{CHF}$, renal/adrenal insufficiency due to possible hypervolemia and sodium retention

- Monitor for hyperkalemia, hypernatremia and accompanying signs and symptoms

- Monitor calcium level, elevated phosphorus levels can cause hypocalcemia

\section{References}

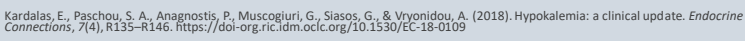
Kear. T. M. (2017). Fulid and Electrolyte Management Across the Age Continuum. Nephrology Nursing Journal: Journal of The American
Nephrology Nurses' Association, 44(6), 491-496. Magnesium - Health Professional Fact Sheet (nih.gov) McPhee, S. J., Papadakis, M. A., \& Rabow, M. W. (2014). Current medical diagnosis \& treatment 2014. 53rd ed. New York: McGraw-Hill Medical. Morton, P. G., Fontaine, D. K. (2018). Critical care nursing: A holistic approach.

Norris, T. L. (2019). Porth's pathophysiology: Concepts of altered health states. (10th ed.). Philadelphia, PA: Wolters Kluwer Health/Lippincott
Williams \& Wilkins.

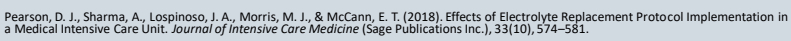
Phosphorus - Health Professional Fact Sheet (nih.gov)

Potassium - Health Professional Fact Sheet (nih.gov)

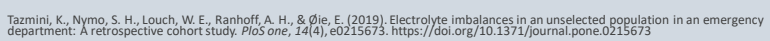

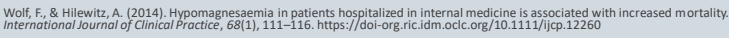




\section{Appendix D}

\section{Research Project Pre-Test/Post-Test}

We are conducting a survey to gather information on medical surgical registered nurses' knowledge about the treatment, management and care of adult patients with electrolytes imbalances. You will be asked to complete a survey prior to your participation in the education session and again at the end. Your participation in either survey is completely voluntary and in no way affects your employment at the Providence VA Medical Center. Your answers will be kept confidential, and no identifiable information will be collected. While the findings from this study may eventually be published, they would be presented based on all of those who answered the questions, not by reporting any individual set of responses.

Please circle your role on nursing unit 6B: NA LPN RN

1. Potassium is the most abundant intercellular cation in the body and plays a role in multiple body functions which include:
A. Acid-Base balance
B. Muscle contraction
C. Conduction of nerve impulses
D. All of the above

2. Magnesium and Potassium have an inverse relationship.
A. True
B. False

3. The clinical manifestations of a magnesium deficiency include:
A. Numbness, muscle weakness, fatigue
B. Increased appetite, weight gain,
C. Nausea, vomiting, diarrhea
D. Shortness of breath, fever, cough

4. The current recommendations for the management of a magnesium level of 1.5 $\mathrm{mEq} / \mathrm{L}$ includes:
A. $2 \mathrm{~g}$ dose intravenously, check levels 4 hours after administration
B. No replacement is required
C. $2 \mathrm{~g}$ dose intravenously, check levels with morning labs
D. $4 \mathrm{~g}$ intravenously, check levels 4 hours after administration 
5. A serum phosphorus level of $3.5 \mathrm{mEq} / \mathrm{dL}$ requires replacement.
A. True
B. False

6. A patient was admitted to your unit and you are reviewing the morning lab results. The patient has a serum $\mathrm{K}+$ level of $3.3 \mathrm{mEq} / \mathrm{L}$, the recommended $\mathrm{K}+$ replacement for this patient would be:
A. $20 \mathrm{mEq}$ orally, recheck level with AM labs
B. $40 \mathrm{mEq}$ intravenously, recheck level with AM labs
C. $60 \mathrm{mEq}$ orally/intravenously, recheck level 4 hours after administration
D. No replacement is required.

7. Intravenous phosphate replacement should be given slowly over four to six hours.
A. True
B. False

8. The maximum infusion rate for intravenous potassium through a peripheral line is:
A. $40 \mathrm{mEq} /$ hour
B. $20 \mathrm{mEq} /$ hour
C. $10 \mathrm{mEq} / \mathrm{hour}$
D. $30 \mathrm{mEq} /$ hour

9. The morning labs have resulted for your patient, indicating a potassium level of $2.9 \mathrm{mEq} / \mathrm{L}$ and a magnesium level of $1.5 \mathrm{mEq} / \mathrm{L}$. The patient is unable to take medications by mouth, and the provider has ordered intravenous replacement of both potassium and magnesium. In what order should you administer the medication to best correct the electrolyte imbalance in this patient?
A. Potassium, Magnesium
B. Dual Administration
C. Magnesium, Potassium
D. No specific order of administration is recommended

10. Electrolytes should be administered cautiously in patients with multiple comorbidities, as well as patients admitted with specific acute conditions which include:
A. ESRD
B. Diabetic Ketoacidosis
C. Chronic Heart Failure
D. All of the Above 